\title{
COLLISIONAL DAMPING OF LANGMUIR WAVES IN THE COLLISIONLESS LIMIT
}

S. P, AUERBACH

\section{PLASMA PHYSICS LABORATORY}

\section{MASTER}

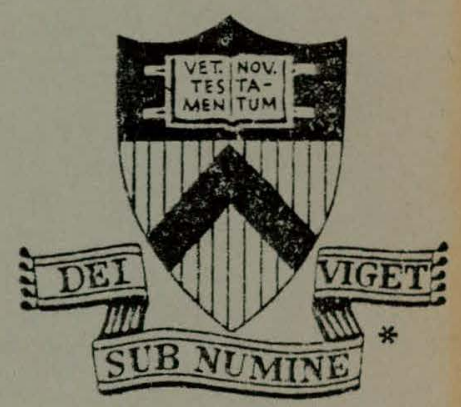

\section{PRINCETON \\ UNIVERSITY PRINCETON, NEW JERSEY}

This work was supported by U. S. Energy Research and Development Administration Contract $E(11-1)-3073$. Reproduction, translation, publication, use and disposal, in whole or in part, by or for the United States Government is permitted. 


\section{DISCLAIMER}

This report was prepared as an account of work sponsored by an agency of the United States Government. Neither the United States Government nor any agency Thereof, nor any of their employees, makes any warranty, express or implied, or assumes any legal liability or responsibility for the accuracy, completeness, or usefulness of any information, apparatus, product, or process disclosed, or represents that its use would not infringe privately owned rights. Reference herein to any specific commercial product, process, or service by trade name, trademark, manufacturer, or otherwise does not necessarily constitute or imply its endorsement, recommendation, or favoring by the United States Government or any agency thereof. The views and opinions of authors expressed herein do not necessarily state or reflect those of the United States Government or any agency thereof. 


\section{DISCLAIMER}

Portions of this document may be illegible in electronic image products. Images are produced from the best available original document. 
NOTICE

This report was prepared as an account of work sponsored by the United States Government. Neither the United States nor the United States Eriergy Research and Development Adninistration, nor any of their employees, nor any of their contractors, subcontractors, or their employees, makes any warranty, express or implied, or assumes any legal liability or responsibility for the accuracy, completeness or usefulness of any information, apparatus, eroduct or process disclosed, or represents that its use would not infringe privately owned rights.

Printed in the United States of America. Available from

National Technical Information Service

U. S. Department of Commerce 5285 Port Royal Road

Springfield, Virginia 22151

Price: Printed Copy \$ $\$$; Microfiche $\$ 3.00$

*Pages

$$
\begin{gathered}
1-50 \\
51-150 \\
151-325 \\
326-500 \\
501-1000
\end{gathered}
$$

NTIS

Selling Price

$\$ 4.00$

5.45

7.60

10.60

13.60 
Collisional Damping of Langmuir Waves in the Collisionless Limit

\author{
S. P. Auerbach
}

Princeton Plasma Physics Laboratory

Princeton University, Princeton, N. J. 08540

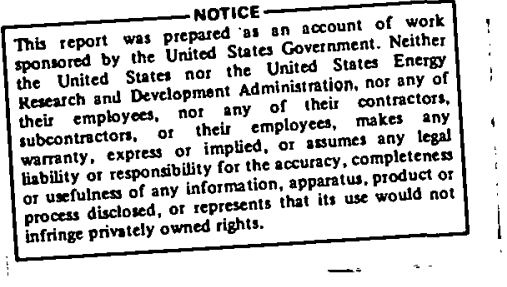

PPPL-1339

April 1977 


\title{
Collisional Damping of Langmuir Waves in the Collisionless Limit
}

\author{
S. P. Auerbach \\ Plasma Physics Laboratory, Princeton University \\ Princeton, New Jersey 08540
}

\begin{abstract}
Linear Langmuir wave damping by collisions is studied in the limit of collision frequency $v$ approaching zero. In this limit, collisions are negligible, except in a region in velocity space, the boundary layer, centered about the phase velocity. If the ratio $\kappa=$ (collisional equilibration time in the boundary layer). (Landau damping time) is small, the boundary layer width scales as $v^{1 / 3}$, and the perturbed distribution function scales as $v^{-1 / 3}$. The damping rate is thus independent of $v$, although essentially all the damping occurs in the collision-dominated boundary layer. Solution of the Fokker-Planck equation shows that the damping rate is precisely the Landau (collisionless) rate. The damping rate is independent of $\kappa$, although the boundary layer thickness is not.
\end{abstract}




\section{Introduction}

The Coulomb collision frequency $v$ in most plasmas is typically qư̈ite small compared to a Langmuir wave frequency $\omega:$

$$
\frac{v}{\omega} \sim \frac{\ln \Lambda}{\mathrm{N}_{\mathrm{D}}}\left(\frac{\mathrm{v}_{\mathrm{T}}}{\mathrm{v}_{\mathrm{f}}}\right)^{3},
$$

where $\ln \Lambda$ is: the Coulomb logarithm, $v_{T}$ is the electron thermal velocity, $v_{f}$ is the wave phase velocity, and $\mathrm{N}_{D}$ is the number of particles in a Debye sphere. Since $\mathrm{N}_{\mathrm{D}}$ is usually quite large, and $\dot{v}_{\dot{f}}$ is usually larger than $v_{T}$ (to avoid heavy damping) $v / \dot{\omega} \ll 1$.

This being so, one frequently sets $\nu=0$ and calculates as if collisions were completely absent. However, a number of somewhat"disturbing problems arise in the course of such calculations. For example, consider the damping of a small-amplitude electrostatic wave (Langmuir wave) with potential

$$
\Phi=\Phi_{0} \cos (k x-\omega t)
$$

Solving the icollisionless Boltzmann equation (i.e., the Vlasov equation) gives as the perturbed distribution function

$$
f_{i}:(x, v, t)=\frac{-e k \Phi_{o}}{\omega-k v} \frac{\partial f_{o}}{\partial v}(v) \cos (k x-\omega t),
$$


where $f_{O}(v)$ is the unperturbed electron distribution function, e is the electron charge, and the ions are assumed to be immobile. There are two obvious deficiencies in this solution, both very well known. First, $f_{l}$ is singular at the phase velocity $v_{f}=\omega / k$. Second, $f_{1}$ is in phase with $\Phi$, and thus the current density $\vec{J}$ is out of phase with the electric field E. Therefore, $\vec{J} \cdot \vec{E}$ vanishes when averaged over one wavelength, and there is apparently no damping!

These two related difficulties have been extensively discussed in the literature, ${ }^{1-6}$ in the context of the purely collisionless theory. However, it has not been emphasized that collisional effects will smooth out singular distributions like Eq. (3). Hence, no matter how small the collision frequency, collisions may not be ignored for particles sufficiently close in velocity to $v_{f}$. Moreover, since collisions are spatially local (the collision operator has no spatial derivatives), they generate terms in $f_{1}$ which are in phase with $\vec{E}$ and cause damping of the wave.

Tu crudely estimate the effect of velocity space diffusion for very small collision frequency $v$ it seems reasonable to insert the collisionless $f_{1}$ into the diffusion part of the collision operator:

$$
\frac{\partial f}{\partial t} \mid \begin{aligned}
& \text { velocity } \\
& \text { diffusion }
\end{aligned}=v v_{T}^{2} \frac{\partial^{2} f}{\partial v^{2}}
$$

where $\mathrm{v}_{\mathrm{T}^{\prime}}$ the electron thermal velocity, is the step size per 
collision. "The "slowing down" term in the collision operator may be neglected near $v=v_{f}$ since it involves only a first derivative in $\left.v_{.}\right)$This leads to the estimate

$$
\left.\therefore \quad \frac{\partial f}{\partial t}\right|_{\text {collision }} \sim \frac{v}{\left(v-v_{f}\right)^{3}} .
$$

Thus, even for arbitrarily small $v$, one might expect to find a "boundary "layer" in velocity space, centexed at $v=v_{f}$ ": with width scaling as $v^{1 / 3}$, in which collisional effects are important.

A more priecise discussion of this boundary layer requires the Fokker-Planck equation, and will be carried out in section II of this paper. The results of this investigation may be summarized as follows. In the limit as $v$ approaches zero. collisions are negligible except in a boundary layer centered at $v_{f}$. Outside the boundary layer the distribution function joins smoothily onto the collisionless distribution. If the ratio $k=$ (collisional equilibration time in the boundary layer) : (Lanidau damping time) is small the boundary layer width scales as $v^{1 / 3}$ and the perturbed distribution function scales as $1 / \hat{j}^{1 / 3}$. [ $k$ is defined following Eq (23). $]$ The damping, which is due solely to collisions, is thus independent of $v$. Integration of the solution to the Fokker-Planck equation'yields the damping rate, which turns out to " be precisely the Landau (collisionless) rate. If $k$ is not small the scaling of the boundary layer thickness and perturbed distribution function is changed, but the 
damping rate is still the Landau rate. Also, for any $k$ the dielectric constant $\varepsilon(k, \omega)$ calculated taking into account the boundary layer is identical to that calculated by interpreting the singularity in Eq. (3) by the Landau prescription, i.e., integrating below the pole at $v=v_{f}$.

The only major difference between the collisional and collisionless theory is that, in the collisional theory, entropy is generated in the boundary layer. For small $v$ the rate of entropy production is independent of $v$. If the background distribution function $f_{0}$ is a Maxwellian, the relation $d Q<$ TdS is obeyed, where $(d Q / d t)$ is the rate at which electrons absorb energy from the waves. In other words, as the wave damps the electrons are irreversibly heated.

Some of the issues treated in this paper have been discussed already in the literature. Lenard and Bernstein, 7 using a model collision operator, have considered the limit as $v$ approaches zero of their exact solution to the Fokker-Planck equation, and recovered the collisionless damping rate. Their first step was to Fourier transform in velocity, so all traces of the boundary layer are hidden in their calculation. Oberman and $\mathrm{Su}^{8}$ in the context of a discussion of plasma echoes, derived several resulls identical to those presented here. However, they did not include the effects of the damping; effectively they set $\kappa=0$. Collisional effects on the quasilinear evolution of Langmuir waves in a collisional plasma were described by Johnston ${ }^{9}$ and Duyle, Denavit 


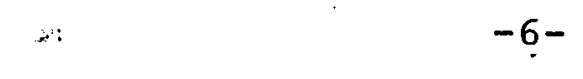

and Hirsch. 10 Johnston notices the dominant effects of collisions within a $v^{1 / 3}$ boundary layer. Dum ${ }^{11}$ has noted phenomena similar to those described in this paper, in the context of the interaction of electrons with a low phase: velocity turbulent spectrum.

\section{Damping and the Boundary Layer}

A damped electrostatic wave, with potential ${ }^{12}$

$$
\Phi=\Phi_{0} e^{-\gamma t} \cos (k x-\omega t)
$$

(where $\omega$ is real) is assumed to be propagating through $a$ uniform medium composed of electrons with charge e, mass.m, and a neutralizing background of immobile ions with number density $\mathrm{n}$ ions per $\mathrm{cm}^{3}$. At time $\mathrm{t}$ the number of electrons within a volume $d^{3} x$, in a velocity region $d^{3} v$, is $n f(x, v, t) d^{3} x d^{3} v$. The phase velocity $v_{f}=\omega / k$ will be assumed large compared to the electron thermal velocity $\mathrm{v}_{\mathbf{T}}$. Transforming to the "wave frame": moving at velocity $v=v_{f}$, and specializing to electrons whose velocity is close to $v_{f}$, the Fokker-Planck equation becomes, as discussedi in Appendix I,

$$
\frac{\partial f}{\partial t}+v \frac{\partial f}{\partial x}-\frac{e}{m} \frac{\partial \Phi}{\partial x} \frac{\partial f}{\partial v}=\nu \frac{\partial}{\partial v}\left[v_{T}^{2} \frac{\partial f}{\partial v}+\left(v+v_{f}\right) f\right] \text {. }
$$

In Eq. (7) $f$ is the distribution function integrated over directions perpendicular to the wave propagation direction, 
$v$ is the velocity relative to $v_{f}$, and $v$ is the collision frequency. Zakharov and Karpman ${ }^{13}$ introduced a convenient set of dimensionless variables, which will be used here: $u=v / v_{T^{\prime}}$ $\alpha=v / v_{f} ; y=k x, \phi_{O}=e \Phi_{O} / T, \mu=v / k v_{T^{\prime}}$ where $\mathrm{T}=\mathrm{mv}_{\mathrm{T}}^{2}$ is the electron temperature in the unperturbed state. Both $\phi_{0}$, the dimensionless wave amplitude, and $\mu$, the dimensionless collision frequency, are assumed to be small. Linearizing by assuming $\mathrm{f}=\mathrm{f}_{\mathrm{O}}+\phi_{\mathrm{O}} \mathrm{f}_{1}$, where $\mathrm{f}_{\mathrm{O}}$ is a background Maxwe $\perp$ ian with temperature $\mathrm{T}$, introducing the operator $\mathrm{L}$,

$$
L=\frac{\partial^{2}}{\partial u^{2}}+\frac{\partial}{\partial u}(u+\alpha),
$$

and assuming $f_{1}$ has time dependence exp $(-\gamma t), E q \cdot$ (7) becomes

$$
-\delta f_{1}+u \frac{\partial f_{1}}{\partial y}-\mu L f_{1}=-\sin y \frac{\partial f_{o}}{\partial u}
$$

where $\delta=\gamma / \mathrm{kv}_{\mathrm{T}}$. If, in addition to $\mu$ being small, it is also true that $\mu \mathrm{Lf}_{1}$ is small, then Fq. (10) has the solution

$$
\begin{aligned}
\mathrm{f}_{1} & =A(u) \sin y+B(u) \cos y, \\
A(u) & -\frac{\delta}{u^{2}+\delta^{2}} \frac{\partial f_{0}}{\partial u}+o(u), \\
B(u) & =\frac{u}{u^{2}+\delta^{2}} \frac{\partial f_{0}}{\partial u}+o(u) .
\end{aligned}
$$

The forms for $A$ and $B$ above exhibit resonance broadening, 
i.e., the damping of the wave shifts the singularity at $\mathrm{v}=\mathrm{v}_{\mathrm{f}}$ away from the real axis. These solutions are not the whole story, however. The rate at which electrons absorb energy from:the wave, averaged over a wavelength, is easily shown to be

$$
\frac{d W}{d t}=\frac{1}{2} n k \alpha T v_{T}^{2} \phi_{0}^{2} \int_{-\infty}^{+\infty} A(u) d u
$$

whish is obviously negative for the $A(u)$ in equation (11). since $d W / d t$ negative corresponds to growth, rather than damping, there is clearly a missing ingredient. In Landau's!' initial value approach ${ }^{2}$ the required change of sign is justified by noting that, although $B+i A$ has a pole in the lower half plane, one must integrate below the pole, rather than above it as in Eq. (12). In the present approach collisions must supply :the missing ingredient: it must be that $\mu \mathrm{Lf}_{1}$ is not negligible near $u=0$.

Near $u=0$ the slowing down term in the collision oper-. ator (second term on the right side of Eq. (7)) may be neglected, sinçe it involves a single velocity derivative and is therefore smaller (by one power of the dimensionless boundary layer thickness) than the diffusion term. This: approximation may be checked a posteriori. Writing, near $\mathrm{u}=0$,

$$
f_{: 1}=[\tilde{A}(u) \sin y+\tilde{B}(u) \cos y]\left[\begin{array}{ll}
\frac{\partial f_{0}}{\partial u} & (0)
\end{array}\right] \text {, }
$$


and neglecting the variation of $\partial f_{o} / \partial u$ (which again may be justified a posteriori, on the grounds that the boundary layer is small), it foliows that

$$
\begin{aligned}
\delta \tilde{A}+\tilde{u B}-1 & =-\mu \frac{d^{2} \tilde{A}}{d u^{2}}, \\
-\delta \tilde{B}+u \tilde{A} & =\mu \frac{d^{2} \tilde{B}}{d u^{2}} .
\end{aligned}
$$

For the moment, assume that $\delta$ is much smaller than 1 , with the precise meaning of "much smaller" to be specified shortly. Equation (14) then becomes

$$
\begin{aligned}
u \tilde{B}-1 & =-\mu \frac{d^{2} \tilde{A}}{d u^{2}}, \\
u \tilde{A} & =\mu \frac{d^{2} \tilde{B}}{d u^{2}} .
\end{aligned}
$$

All reference to the collision frequency in Eq. (15) may be removed by the scalings $u=\mu^{1 / 3} \zeta,[\tilde{A}(u), \tilde{B}(u)]=\mu^{-1 / 3}[a(\zeta)$, $\mathrm{b}(\zeta)]$; Eq. (15) is then

$$
\begin{aligned}
\zeta b-1 & =-a^{\prime \prime}, \\
\tau a & =b^{\prime \prime},
\end{aligned}
$$

where "' " means $\mathrm{d} / \mathrm{d} \zeta$.

The solutions to Eq. (16) must match smoothly onto the collisionless theory with $O(\mu)$ corrections, given in Eq. (1I). 
Since $\delta$ has'been neglected in Eq. (16), b( $\zeta)^{\prime}$ must match onto

$$
B(u)=\frac{l}{u} \frac{\partial f_{0}}{\partial u} .
$$

Thus, $b(\zeta)$ has asymptotic behaviour

$$
b(\zeta) \sim \frac{\mu^{1 / 3}}{f_{0}^{\prime}} B=\frac{1}{\zeta} .
$$

The asymptotic behaviour of a $(\zeta)$ matches onto the $O(\mu)$ term in Eq. (11):

$$
A(u)=\frac{\mu}{u} L B,
$$

and therefore

$$
a(\zeta) \sim \frac{2}{\zeta^{4}}
$$

The crux of the matter is that Equations (16) and the conditions on the asymptotic behaviour of $a(\zeta)$ and $b(\zeta)$ are independent of $\mu$. The matching described above therefore occurs at a value of $\zeta$ which is independent of $\mu$, and thus at a value of $u$ which scales as $\mu^{1 / 3}$. In other words, there is a boundary layer whose thickness is proportional to $\mu^{i / 3}$. Since the perturbed distribution function scales as $\mu^{-1 / 3}$ within the boundary layer, the damping rate [see Eqs. (12): and (34)] is independent of collision frequency. 
It is convenient to combine $a(\zeta)$ and $b(\zeta)$ into a new function $E=b+i a$, which satisfies the differential equation

$$
\zeta E=i E "+1
$$

It is shown in Appendix II that the solution to Eq. (21) which matches onto the asymptotic behaviour given in Eqs. (18) and (20) is $^{14}$

$$
F(\zeta)=-i \int_{0}^{\infty} \exp \left(\frac{-z^{3}}{3}+i \zeta z\right) d z
$$

A graph of the functions $a(\zeta)$ and $b(\zeta)$ is given in Fig. 1 . The change in sign of $a(\zeta)$ at $\zeta \sim \pm 2.5$ should be noted. This sign change is needed to make the integral in Eq. (12) positive. Fig. 2 illustrates how the boundary layer behaviour

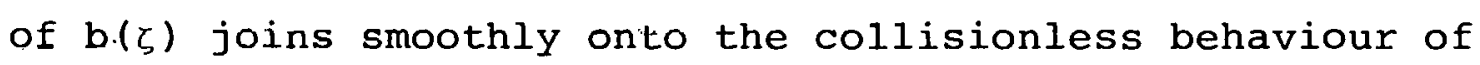
$B(u)$.

If the assumption that $\delta$ is negligible is dropped, scaling $u, A$ and $B$ as above produces

$$
\begin{aligned}
& \kappa a(\zeta)+\zeta b(\zeta)-1=-a^{\prime \prime}(\zeta), \\
& -\kappa b(\zeta)+\zeta a(\zeta)=b^{\prime \prime}(\zeta),
\end{aligned}
$$

where $k=\delta / \mu^{1 / 3}$. This is equivalent to Eq. (16) if $|\kappa| \ll 1$. 
$1:$

Apparently, the previous assumption $\delta<1$ meant, more precisely, $\delta<\mu^{1 / 3}$. In fact, $k$ is more likely to be large, rather than small, since $\mu$ is quite small. (Recall that $\kappa=\delta / \mu^{1 / 3}$, where $\delta=\gamma / \mathrm{kv}_{\mathrm{T}^{\prime}}$, and $\left.\mu=\nu / \mathrm{kv}_{\mathrm{T}}.\right)$ If $\kappa$ is not small, then the $\mu$-independence exhibited by Eq. (16) is not present in Eq. (23) and consequently the $\mu^{1 / 3}$ scaling of the boundary layer is not valid.

Despite their differences, Eqs. (23) can be effectively transformed into Eqs. (16) by introducing $\xi=\zeta-i_{k} ;$ Eq. (33) bernmes

$$
\xi E=i E^{\prime \prime}+1
$$

where "'" means $d / d \xi$. The appropriate asymptotic behaviour follows from Eq. (11): $E \sim 1 / \xi$. The solution ${ }^{9}$ to Eq. (24) which matches onto this behaviour is identical to Eq. (22):

$$
\therefore E(\xi)=-i \int_{0}^{\infty} \exp \left(\frac{-z^{3}}{3}+i \xi z\right) d z \text {. }
$$

The sole effect of the damping term is to depress the variable $\zeta$ in the complex plane. ${ }^{15}$ Graphs of $a(\zeta)$ and $b(\zeta)$ calculated from Eq. (25) are shown in Fig. 3 for various values of . $k$. The thickness of the boundary layer is a somewhat $\underset{i}{a}$ bitrary quantity. It may be defined precisely by requiring that the real and imaginary parts of $\mathrm{E}$ be sufficiently close (say, within 5\%) to the appropriate values derived from the coilisionless theory with $O(\mu)$ corrections, viz., $E=1 / \xi$ if $\kappa \neq 0$, 
or $a(\zeta)=2 / \zeta^{4}, b=1 / \zeta$ if $k=0$. Clearly, the boundary layer thickness is related to the asymptotic behaviour of $\mathrm{E}(\xi)$. A simple analysis shows that the complex $\xi$ plane is divided into two regions, as shown in Fig. 4. In the shaded region, which corresponds to $|\zeta|<\kappa \sqrt{3}, k>0$, as $|\xi|$ becomes large, $\mathrm{E}$ behaves as

$$
E(\xi) \sim \frac{-i \sqrt{\pi}}{(\kappa+i \zeta)^{1 / 4}} \exp \left[\frac{2}{3}(\kappa+i \zeta)^{3 / 2}\right]
$$

Outside the shaded region, as $|\xi|$ becomes large,

$$
E \sim \frac{1}{\xi}+\frac{2 i}{\xi}+\cdots \cdot \cdot
$$

For $k \leq 0$, as $\zeta$ varies the shaded region is never encountered and thus if either $k$ or $\zeta$ is large $E$ will be close to its asymptotic value. For example, with $\kappa=0$, for $|\zeta| \geqslant 8$, numerical work shows that both $a(\zeta)$ and $b(\zeta)$ are within five percent of the asymptotic values $a=2 / \tau^{4}, b=1 / r$, For $\kappa=-5$, even at $\zeta=0$ both $a(\zeta)$ and $b(\zeta)$ are within five percent of the asymptotic values given by $\mathrm{E}=1 / \xi$. If $K$ is positive, the behaviour given by Eq. (26) prevails until $\zeta$ leaves the shaded region, i.e., until $|\zeta|>\kappa \sqrt{3}$. If $\kappa$ is not too large, say, $k \leqslant 2$, it is still found numerically that if $|\zeta| \geqslant 8$, $a(\zeta)$ and $b(\zeta)$ are within five percent of the values determined from $E \sim 1 / \xi$. On the other hand, if $\kappa$ is large, then the transition over to the collisionless behaviour only occurs for $|\zeta| \geqslant k \sqrt{3}$. 
Assembling the above remarks, a simple picture of the boundary layer emerges. The boundary layer width is determined by the ratio $k=\gamma / \nu^{1 / 3}\left(k v_{T}\right)^{2 / 3}$. It will be shown below that $\gamma$ is precisely the Landau damping rate, so the parameter $\kappa$ may be varied by varying either $v_{f} / v_{T^{\prime}}$ the slope $\partial f_{o} / \partial v$ of the unperturbed distribution function, or the collision frequency $v$. If $\partial f_{0} / \partial v>0$, as in a bump-on-tail type of instability, $k$ will be negative, while for a Maxwellian distribution $\kappa$ is positive. For $k$ sufficiently nogative, say $k \leq-5$, the distinction between the boundary layer and the rest of velocity space disappears. The collisionless behaviour $E \sim L / \xi$ is a good approximation to $E(\xi)$ for all real values of velocity. In other words, as seen from the real axis, the distribution function appears to have a pole above the real axis, just as j.t would in the collisionless theory. Of course $E$ has no polfs; the approximation $E \sim 1 / \xi$ breaks down for small $\dot{\xi}$. If $k$ is neither too positive nor too negative, say $-5 \leq \kappa \leq 2$, there is a boundary layer given roughly by $|\zeta| \leq 8$. In terms of the fixed frame velocity $v$ this means

$$
\left|\frac{\mathrm{v}_{\mathrm{T}} \mathrm{v}_{\mathrm{f}}}{\mathrm{v}_{\mathrm{T}}}\right| \leq 8 \mu^{1 / 3}
$$

If $k$ is large, say $k>2$, the boundary layer thickness is determined by $|\zeta|<k \sqrt{3}+c_{j}^{*}$ the constant $c$ taking into account that the transition between the behaviours given in Eqs. (26) and (27) does not occur. instantaneously. In the fixed 
frame $|\xi| \leq \kappa \sqrt{3}+C$ reads

$$
\left|\frac{v-v_{f}}{v_{T}}\right| \leqslant \frac{\gamma}{k v_{T}} \sqrt{3}+\text { constant }
$$

Within the boundary layer $\mathrm{E}(\xi)$ is very large and highly oscillatory. This behaviour is shown analytically by Eq. (26) and numerically in Fig. 3 .

The physical significance of the parameter $\kappa$ may be $e$ ?ucidated as follows. Within the boundary layer an estimate of the typical time $\tau_{\text {eq }}$ for collisions to produce equilibrium is

$$
\begin{aligned}
\left.\frac{E}{\tau} \backsim \frac{\partial f}{\partial t}\right|_{\text {collisions }} & =\frac{\nu}{\mu^{2 / 3}} \frac{\partial^{2} f}{\partial \zeta^{2}} \\
& \sim \frac{\nu}{\mu^{2 / 3}}(\zeta E-1),
\end{aligned}
$$

where the last estimate follows from Eq. (24). For moderate sized values of $\zeta, \zeta \mathrm{E}$ is of order $\mathrm{E}$, and thus $\mathrm{T}$ eq scales as $\tau_{\text {eq }} \sim \mu^{2 / 3} / v$. (Larger values of $\zeta$ give rise to smaller estimates of $\tau_{\text {eq' }}$ but presumably the slowest process determines the overall rate.) The ratio of $\tau$ eq to the damping time $\tau=1 / \gamma$ is

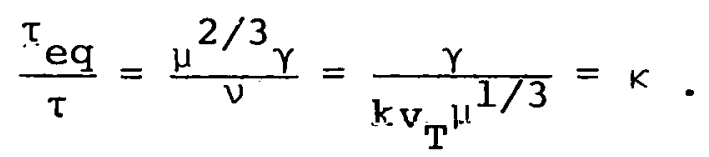

If $K$ is large, the wave damps too rapidly for collisions to cause equilibration. This is why $E$ is so oscillatory for larye $k$. 


\section{III: The Dielectric Constant and Damping Rate}

In a dispersive medium with dielectric constant $\varepsilon(k, \omega)$, the energy of a wave, averaged over one wavelength, is ${ }^{16}$

$$
\omega \frac{\partial \varepsilon^{\prime}}{\partial \omega} \frac{<E^{2}>}{8 \pi}
$$

where the brackets denote averaging and $\varepsilon^{\prime}=$ Rer. Energy conservation implies that the damping decrement $\gamma$ is given by

$$
2 \gamma \omega \frac{\partial \varepsilon^{\prime}}{\partial \omega} \frac{\left\langle E^{2}>\right.}{8 \pi}=\frac{d W}{d t}
$$

where dW/dt, given in Eq..(12), is the rate at which electrons gain energy from the wave. Thus,

$$
\gamma=\left(\omega \frac{\partial \varepsilon}{\partial \omega}\right)^{-1} \frac{\ddot{\omega}_{\mathrm{p}}^{2} \alpha}{k} \int_{-\infty}^{+\infty} \mathrm{du} A(u)
$$

The dielectric constant is $\varepsilon=1+(4 \pi i \sigma / \omega)$ where $\sigma$ is the conductivity: $J=U E$. Expressing $J$ in terms of $f_{1}$, the real and imaginary parts $\sigma_{R}$ and $\sigma_{I}$ of the conductivity are, in the wave frame:

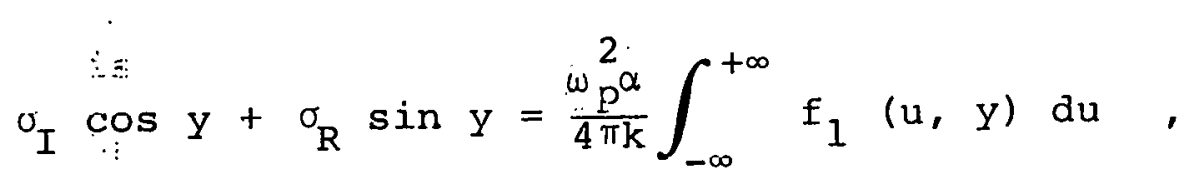

(recall that $\left.\mathrm{f}_{\mathrm{I}}=\mathrm{A}(\mathrm{u}) \sin \mathrm{y}+\mathrm{B}(\mathrm{u}) \cos \mathrm{y}\right)$.

The integral in Eq. (35): is to be evaluated using the functions given in Eq. (il) oütside the boundary layer and 
and $\mathrm{E}(\xi)$ inside the boundary layer. It follows from Eq. (25) that $E(\xi)^{*}=-E\left(-\xi^{\star}\right)$; hence $a(\zeta)$ is an even function of $\zeta$ and $b(\zeta)$ is odd. Because $b(\zeta)$ is odd the boundary layer contribution to $\sigma_{I}$ vanishes, and

$$
\sigma_{I}=\frac{\omega_{\mathrm{p}}^{2}}{4 \pi \mathrm{k}} \int^{\prime} d u \frac{\mathrm{u}}{\mathrm{u}^{2}+\delta^{2}} \frac{\partial \mathrm{f}_{\mathrm{o}}}{\partial \mathrm{u}}+o(\mu)
$$

where the " " indicates that the integration region exclucies the boundary layer. Since the boundary layer is very thin, this is essentially a principal value integral. To calculate $\sigma_{R}$ note that $A(u)$ decreases rapidly away from $u=0$. Thus, $\partial f_{0} / \partial u$ may be set equal to $\partial f_{0} / \partial u(u=0)$ when doing the velocity integrals in Eq. (35). Neglecting terms of order $\mu$, the asymptotic behaviour of $a(\zeta)$ joins onto the behaviour of A(u) outside the boundary layer. The contributions from inside and outside the boundary layer need not be separated. Now,

$$
A(u) d u=\frac{\partial f_{0}}{\partial u}(0) a(\zeta) d \zeta,
$$

therefore,

$$
\sigma_{R}=\frac{\omega_{p}^{2} \alpha}{4 \pi k} \frac{\partial f_{o}}{\partial u}(u=0) \operatorname{Im} \int_{-\infty}^{+\infty} E\left(\zeta-i_{k}\right) d \zeta+o(\mu)
$$

This integral is evaluated in Appendix II for $k=0$. However, $\mathrm{E}$ is an analytic function; the integral is independent of $\mathrm{K}$. 
Therefore,

$$
\sigma_{R}=\frac{\ddot{\omega}_{p}^{2} \dot{p}}{4 \pi k}\left(\frac{\pi \partial f_{0}(0)}{\partial u}\right)+O(\mu)
$$

and the dielectric constant is

$$
F=I-\frac{\omega p^{2} \alpha}{\omega k}\left(P \int_{-\infty}^{+\infty} d u \frac{u}{u^{2}+\delta^{2}} \frac{\partial f_{o}}{\partial u}+i \pi \frac{\partial f_{o}(0)}{\partial u}\right)+O(\mu)
$$

This is identical to the dielectric function $\varepsilon_{C}$ of a collisionless plasma

$$
\varepsilon \dot{c}(k, \omega)=1+\frac{\omega p^{2}}{k} \int d v \frac{1}{\omega-k v} \frac{\partial \dot{f}_{o}}{\partial v},
$$

evaluated at: the complex frequency $\omega-i \gamma$. The imaginary. contribution $i \pi \mathrm{E}_{0}^{\prime}$ in Eq. (40) arises in the collisionless theory via Landau!s prescription ${ }^{2}$ to integrate below the pole at $\omega=\mathrm{kv}$.

Now that $f(k, w)$ is known, the damping rate may be calculated, using.Eq. (34). Alternatively, it may be calculated by solving $\varepsilon(k, \omega-i \gamma)=0$. The result, by either method; is that $\gamma$ is prëcisely the Landau (collisionless) rate

$$
\gamma=\gamma_{\text {Landau }}=\frac{-\pi}{2} \omega \cdot \frac{\omega p^{2}}{k^{2}} \frac{\partial f}{\partial v}\left(v=v_{f}\right)
$$

It should be noted that the contribution of the region outside the boundary layer to $y$ is negative. The ratio of the magnitude of this contribution to the boundary layer 
contribution depends on $k$, and varies from zero for $k=0$ to $1 / 4$ for large $k$. For any value of $k$, most of the damping occurs within the boundary layer, i.e., within the region where collisional effects are dominant.

It is perhaps surprising that the damping occurs at the collisionless rate, even though most damping takes place within the boundary layer. However, since $f_{1}$ scales as $\mu^{-1 / 3}$ in the boundaly layer, whose thickness scales as $\mu^{1 / 3}$ (at least for small $k$ ), for small $\mu$ the damping rate must be independent of $\mu$. The physically plausible assumption that $\gamma$ is a continuous function of $\mu$ then guarantees that $\gamma=\gamma_{\text {Landau }}$.

\section{Entropy Production}

Entropy is produced by collisions at a rate

$$
\frac{d S}{d t}=-\left.n \int d x d v \frac{\partial f}{\partial t}\right|_{C} \ln f
$$

Ignoring $O(\mu)$ terms, entropy production occurs only in the boundary layer, where

$$
\left.\frac{\partial f}{\partial t}\right|_{C}=v v_{T}^{2} \frac{\partial^{2} f}{\partial v^{2}}
$$

Averaging over one wavelength, and integrating Eq. (43) by parts, 


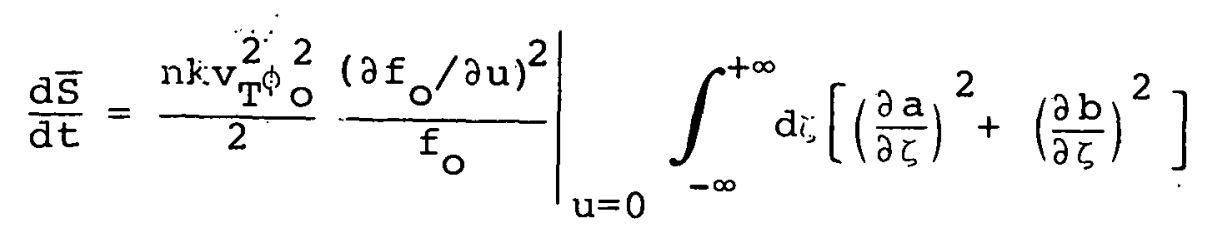

which has no expliait dependence on $\dot{\mu}$. Using Fq. (23)

$$
\begin{aligned}
& \int_{-\infty}^{+\infty}\left[\left(\frac{\partial a}{\partial \zeta}\right)^{2}+\left(\frac{\partial b}{\partial \zeta}\right)^{2}\right] d \zeta=\int_{-\infty}^{+\infty}\left[-a(\zeta)+\kappa\left(a^{2}+b^{2}\right)\right] d \zeta \\
& =\pi+k \int_{-\infty}^{+\infty} \mathrm{d} \zeta\left|\mathrm{E}\left(\zeta-i_{k}\right)\right|^{2} \\
& =\pi\left[1+2^{2 / 3} k \int_{-\infty}^{+\infty} d z \exp \left(\frac{-z^{3}}{3}+2^{2 / 3} k z\right)\right] \text {. }
\end{aligned}
$$

Dividing this by the rate $d w / d t$ at which energy is absorbed by the electrons, assuming that $f_{0}$ is a Maxwellian with temperature $T$, the result is

$$
T \frac{d \vec{S}}{d t}=\frac{d w}{d t}\left[1+2^{2 / 3} \kappa \int_{0}^{\infty} d u \cdot \exp \left(\frac{-u^{3}}{3}+2^{2 / 3} k u\right)\right]>\frac{d w}{d t}
$$

How does this relate to the thermodynamic relation do $\leq$ TdS? setting dQ/dt equal to the rate of energy flow to the electrons, clearly dQ < TdS; i.e.,. in the sense of the second Law of Thermodynamics, Landau damping is an irreversible process. 
As mentioned before, the parameter $k$ is likely to be quite large because the collision frequency is very small. For large $K$ the ratio of the entropy production rate to the heating rate is enormous: $\operatorname{Td} \bar{s} / \mathrm{dW} \sim \kappa^{3 / 4} \cdot \exp \left(4 k^{3 / 2} / 3\right)$. At first glance, it appears paradoxical that this ratio, which in some sense is a measure of irreversibility, should increase so rapidly as the collision frequency decreases (and $\kappa \sim \gamma / \nu^{1 / 3}$ increases). This apparent paradox is resolved by calculating the entropy. Expanding the distribution function to second order, $f=f_{0}+\phi_{0} f_{1}+\phi_{0}^{2} f_{2}$, and averaging over one wavelength, the entropy is

$$
\begin{aligned}
\bar{s} & =-n \int \overline{f \ln f} d v \\
& =\frac{\bar{E}}{T_{o}}-\frac{n \phi_{o}^{2}}{2} \int \frac{\overline{f_{l}^{2}}}{\bar{f}_{o}} d v+\text { const. }
\end{aligned}
$$

where $\overline{\mathrm{E}}$ is the average energy:

$$
\bar{E}=\frac{1}{2} n m \int v^{2}\left(f_{o}+\phi_{o}^{2} \bar{f}_{2}\right) d v
$$

IL may be shown, either by directly solving for $\bar{f}_{2}$, or by invoking energy conservation, that the second order contribution to $\overline{\mathrm{E}}$ is precisely the negative of the electric field energy $E^{2} / 8 \pi$. The secund term in Eq. (49) represents the 
decrease in entropy, or increase in order, due to the presence of the wave. The integral appearing in Eq. (49) is dominated by the boundary layer. Taking into account only the boundary layer

$\bar{s}=\frac{\bar{E}}{T_{o}}-\left[\frac{2^{2 / 3^{2}} \pi n \phi_{o}^{2}}{1 f_{0}}\left(\frac{\partial f_{o}}{\partial u}\right)^{2}\right] \frac{1}{\mu^{1 / 3}}\left[\int_{0}^{\infty} \exp \left(\frac{-u^{3}}{3}+2^{2 / 3} \kappa u\right) d u\right] \exp (-2 \gamma t)$,

where $f_{0}$ and $\partial_{0} / \partial u$ are evaluated at the phase velocity $(u=0)$. If the wave is suddenly set up in the plasma at time $t=0$, the plasma entropy is decreased by a large amount, proportional to $\mu^{-1 / 3} \kappa^{-1 / 4} \exp \left(4 k^{2 / 3} / 3\right)$ if $k$ is large. Note that the entropy decrease required to set up the wave increases rapidly as the collision frequency decreases ( $\mu$ decreases and $k$ increases). As the wave damps away, the distribution function approaches a Maxwellian, and so the entropy must increase to the Maxwellian level. Since the entropy at $t=0$ is very low, compared to a Maxwellian, the rate of entropy increase must be very large to bring the entropy to the Maxwelilian level at $t=\infty$. This: explains why $\mathrm{T} \ddot{d} \bar{s} / d t$ is so much larger than $\mathrm{dW} / \mathrm{dt}$, for large $k$. The argument just given may be rephrased, as follows. The : initial state (wave imposed at $t=0$ ) is highly ordered, for small $\mu$, but the final state (Maxwellian at $t=\infty$ ) has maximal 
disorder. The transition from order to disorder occurs, roughly, in a time of order $1 / \gamma$, which is independent of $\mu$. Thus, for small $\mu$, the rate of entropy production, or destruction of order, must be very large. The heating rate, i.e., the rate at which electric field energy is transferred to the plasma is, of course, independent of $\mu$.

The high degreee of order necessary to set up this wave raises the question of whether any experimental apparatus, which excites a plasma initially in thermal equilibrium, could produce the state described in this paper. This question will be discussed in a forthcoming publication. 


\section{Discussion}

To arrive at a clear physical understanding of the results of this. work, several characteristic frequencies and dimensionless parameters must be kept in mind. In the presence of an electrostatic wave of wavenumber $k$ and dimensionless amplitude $\phi_{0}\left(\phi_{0}=e \Phi_{0} / T, \Phi_{0}\right.$ being the electric potential of the wave) there are two characteristic frequencies. The bounce frequency $\omega_{B}=k v_{T} \sqrt{\phi_{0}}$ is the frequency (as seen in. the wave frame) at which trapped electrons oscillate in the troughs of the wave. The detrapping frequency $\omega_{D}$ is the inverse of the time for a typical trapned electron to be detrapped by collisions. Collisions cause a random walk in velocity space, with mean square velocity change $\Delta v$ in time $\Delta t$ given by $(\Delta v)^{2}=D \Delta t$; where $D=v v_{T}^{2}$. Since $\Delta v$ must be large enough to detrap the ellectron

$$
\frac{1}{2} m(\Delta v)^{2} \sim e_{0}
$$

and therefore $\dot{\omega}_{D}=v / \phi_{O}$. The evolution of the wave is determined by the ratio of detrapping, to bounce frequencies:

$$
\frac{\omega_{D}}{\omega_{B}}=\frac{: \mu}{\phi_{O}^{3 / 2}}
$$

where $\mu=\nu / k v_{T}$ is the dimensionless collision frequency. Zakharov and Karpman ${ }^{13}$ have treated the case where trapped 
particles bounce many times before being collisionally detrapped, i.e., $\phi_{O}^{3 / 2}>\mu$. The opposite limit, $\omega_{B} \ll \omega_{D}$ ' i.e., $\phi_{0}{ }^{3 / 2} \ll \mu$ is the subject of the present work. This is a very collisional regime: particles are collisionally detrapped before they have time to bounce.

Stringer, ${ }^{17}$ in the very analogous case of neoclassical diffusion in the plateau regime, has argued that it is only those particles which, due to collisions, experience a large fractional change in velocity $(\Delta v / v i 1)$ as they traverse one wavelength, that give rise to appreciable damping. Consider the class of particles (analogous to Stringer's "passing" particles) with wave frame velocity $|v| \leq r v_{T}$, where $r$ is to be determined. It takes these particles a time $\Delta t \geq 1 / \mathrm{krv}_{\mathbf{T}}$ to traverse the wave. The condition $\Delta v / v \sim 1$ is then

$$
1 \sim\left(\frac{\Delta v}{v}\right)^{2} \sim \frac{D \Delta t}{r^{2} v_{T}^{2}} \geq \frac{\mu}{r^{3}} .
$$

Thus $r \sim \mu^{1 / 3}$, and collisions are important. only for $\left|v / v_{T}\right| \lesssim \mu^{1 / 3}$. This is the origin of the boundary layer described above. Note that most boundary layer particles are not trapped. Trapped particles have velocity $|\mathrm{v}| \leq \phi_{0}^{1 / 2} \mathrm{v}_{\mathrm{T}}$, and $r v_{T} / \phi_{O}^{1 / 2} v_{T}=\left(\mu / \phi_{O}^{3 / 2}\right)^{1 / 3}>1$.

To estimate the damping, it must be yemembered that collisions conserve energy. They cause damping only by altering the distribution function, thereby causing a current to flow in the fixed frame. In the wave frame, diffusion dominates 
the collision" operator for boundary layer particles, and the Fokker-Planck equation becomes (neglecting the time derivative)

$$
v \frac{\partial f_{1}}{\partial x}-\frac{e}{m} \frac{\partial \phi}{\partial x} \frac{\partial f_{o}}{\partial v}=v v_{T}^{2} \frac{\partial^{2} f_{1}}{\partial v^{2}}
$$

The scale is set by the inhomogeneous term. Estimating $\partial^{2} f_{1} / \partial v^{2} \backsim f_{1} /\left(f v_{T}\right)^{2}$ leads to the order of magnitude estimate

$$
f_{1} \sim \frac{\phi_{0}}{\mu} \frac{\partial f_{0}}{\partial / 3} v_{T} \frac{\partial}{\partial v}
$$

which agrees with the scalings observed in Sec. II. The fixed frame current density is then

$$
\begin{aligned}
\mathrm{J}=\operatorname{nev}_{\mathrm{f}} \int \mathrm{f}_{1} \mathrm{dv} & \sim \operatorname{nev}_{\mathrm{f}} \mu^{1 / 3} \mathrm{v}_{\mathrm{T}^{\mathrm{f}}{ }_{1}} \\
& \sim \omega_{\mathrm{p}}^{2} \Phi_{\mathrm{O}} \mathrm{v}_{\mathrm{T}} \frac{\partial \mathrm{f}}{\partial \mathrm{v}},
\end{aligned}
$$

which is independent of collision frequency $v$. The damping decrement is

$$
\gamma \sim \frac{\vec{J} \cdot \dot{E}}{E^{2}} \sim \frac{\omega \omega_{p}^{2}}{k^{2} \cdot} \frac{\partial f_{o}}{\partial v},
$$

which is, up to numerical factors, the Landau (collisionless) damping rate. As mentioned above, the essential reason that the damping is independent of $v$ is that the boundary layer width scales as $v^{1 / 3}$, while $f_{1}$. scales as $\phi_{0} / \nu^{1 / 3}$. The current 
is independent of $v$, and so is the damping. If the parameter $K$ is large, this simple picture does not apply, because the damping occurs too rapidly compared to collisional equilibration times. However; as shown before, the main effect of $\kappa$ (i.e., of the damping term) is to effectively shift the velocity down into the complex plane. Integrals over the distribution function are, by analyticity, not changed by this shift, and $\gamma$ remains at the collisionless value.

\section{ACKNOWLEDGMENTS}

I would like to thank Drs. A. Boozer, B. Cohen, C. Oberman, E. Ott, M. Mostrom and T. H. Stix for discussions. This work was supported by the Electric Power Research Institute, Inc., Contract No. RP 423 - 1, and United States Energy Research and Development Administration Contract E $(11-1)-3073$. 


\section{Appendix I}

The Fokker-Planck equation may be written ${ }^{18}$

$$
\underset{\partial \dot{f}}{\partial \underline{t}}+\vec{v} \cdot \frac{\partial f}{\partial \vec{x}}-\frac{e}{m} \nabla \Phi \cdot \frac{\partial f}{\partial \vec{v}}=\frac{\partial}{\partial \vec{v}} \cdot \vec{F},
$$

where $\overrightarrow{\mathrm{F}}$ is the velocity space particle filux due to collisions. It vill be assumed that the phase velocity $v_{f}$ is large compared to the thermal velocity, and that $f$ is Maxwellian except for a small region near the phase velocity. This allows the Rosenbluth potentials to be evaluated. For velocities $v>>v_{f}$, the flux becomes ${ }^{13}$

$$
F_{i}=\frac{\Gamma}{v^{3}}\left[v_{i} f+\frac{1}{2}\left(v^{2} \delta_{i j}-v_{i} v_{j}\right) \frac{\partial f}{v_{j}}-\frac{T}{2 m}\left(\frac{\delta_{i j} v^{2}-3 v_{i} v_{j}}{v^{2}}\right) \frac{\partial f}{v_{j}}\right],
$$

where $\mathrm{T}$ is the electron temperature, which is related to the electron thermal velocity by $v_{T}^{2}=T / m$, and

$$
\therefore \quad \quad T^{\prime}=\frac{4 \pi n c^{4} \ln \Lambda}{m^{2}},
$$

where $\ell n \Lambda$ is the Coulomb logarithm. The wave is propagating in the $\mathrm{x}$ direction; $\mathrm{f}$ will be taken as

$$
f=f\left(v_{x}, v_{\perp}\right)
$$

with $v_{\perp}=\left(v_{y}^{2}+v_{z}^{2}\right)^{1 / 2}$. It follows from (I.2) that 


$$
\begin{gathered}
F_{x}=\frac{\Gamma}{v^{3}}\left[v_{x} f+\frac{\partial f}{\partial v_{x}}\left(\frac{v_{\perp}^{2}}{2}+\frac{T}{m}\right)-\frac{v_{\perp} v_{x}}{2} \frac{\partial f}{\partial v_{\perp}}\right] \\
\left.\vec{F}_{\perp}=\frac{\Gamma}{v^{3}} \vec{v}_{\perp} f f+\frac{\partial f}{\partial v_{x}}\left(\frac{-v_{x}}{2}+\frac{3 T}{2 m} \frac{v_{x}}{v^{2}}\right)+\frac{\partial f}{\partial v_{\perp}}\left[\frac{v_{x}^{2}}{v_{\perp}}+\frac{T}{2 m}\left(\frac{3 v_{\perp}}{v^{2}}-\frac{1}{v_{\perp}}\right)\right]\right)^{\prime},
\end{gathered}
$$

where $v^{2}=v_{x}^{2}+v_{\perp}^{2}$, and $\vec{F}_{\perp}$ is the flux in the $y-z$ plane (in velocity space). Assume now that $\vec{F}_{\perp}$ vanishes. This assumption is consistent with the symmetry of the problem, though it is by no means a necessary consequence of that symmetry. In order to understand the structure of the flux $\vec{F}_{\perp}$, it is instructive to insert a Maxwellian $f$ into $\vec{F}_{\perp}$, and inquire why $\overrightarrow{\mathrm{F}}_{\perp}$ vanishes. It turns out that, of the six terms in $\vec{F}_{\perp}$, there are two large terms, viz., $(-i / 2) v_{x} \partial f / \partial v_{x}$ and $(1 / 2)\left(v_{x}^{2} / v_{\perp}\right) \partial f / \partial v_{\perp}$ which cancel against each other. The remaining four terms are smaller by a factor $\left(v_{T}^{2} / v_{f}^{2}\right)$, and cancel among themselves. Even in the presence of the wave the large terms must cancel each other, and thus:

$$
\frac{-v_{x}}{2} \frac{\partial f}{\partial v_{x}}+\frac{v_{x}^{2}}{2 v_{\perp}}=n\left(\frac{v_{T}^{2}}{v_{f}^{2}} f\right)
$$

It follows that

$$
F_{x}=\frac{\Gamma}{v^{3}}\left[v_{x} f+\frac{T}{m} \frac{\partial f}{\partial v_{x}}+o\left(\frac{v_{T}^{3}}{v_{f}^{3}} f\right)\right] \text {. }
$$

Dropping terms of order $\left(v_{T}^{2} / v_{f}^{2}\right)$ and integrating over directions transverse to the wave, the Fokker-Planck equation becomes, 19 for $v$ close to $v_{f}$, 


$$
\begin{gathered}
f=f(x, v) \\
\frac{\partial f}{\partial t}+v \frac{\partial f}{\partial x}-\frac{e}{m} \frac{\partial \Phi}{\partial x} \frac{\partial f}{\partial v}=v \frac{\partial}{\partial v}\left(v_{T}^{2} \frac{\partial f}{\partial v}+v_{f}\right)
\end{gathered}
$$

where

$$
\begin{aligned}
f(x, v) & =\int f\left(x, v_{x}, \vec{v}_{L}\right) \cdot d v_{\perp}, \\
v & =v_{x}, \\
v & =\frac{\Gamma}{v_{f}^{3}} .
\end{aligned}
$$


Appendix II

The equation

$$
\zeta E=i E^{\prime \prime}+1
$$

may be solved by a contour integral technique. Write

$$
E(\zeta)=\int_{C} e^{i z \zeta} f(z) d z
$$

and represent the inhomogeneous term by

$$
1=\int_{C} e^{i z \zeta} \delta(z) d z
$$

where $\delta(z)$ is Dirac's delta-function. Clearly the contour C must pass through the origin. The right side of Eq. (II.1) may be represented as

$$
\begin{aligned}
\zeta E(\zeta) & =-i \int_{C}\left(\frac{d}{d z} e^{i z \zeta}\right) f(z) d z \\
& =i \int_{C} e^{i z \zeta} f^{\prime}(z) d z,
\end{aligned}
$$

provided that $f(z)$ exp izh vanishes at the ends of the contour. Therefore,

$$
f^{\prime}(z)=-z^{2} f(z)-i \delta(z)
$$


If $z \neq 0, f(z)=\varepsilon \exp \left(-z^{3} / 3\right)$. Integrating Eq. (II.5), one finds that $f(z)$ must jump by $-i$ as $z$ crosses the origin. This requirement may be satisfied by setting $\varepsilon=0$ on the segment of $C$ before the origin, and $\varepsilon=-i$ after the origin. The requirement that $\exp \left(i z \zeta-z^{3} / 3\right)$, vanish at the ends of the contour is satisfied provided $\operatorname{Re}^{3}<0$ as $z \rightarrow m$. Thus,

$$
E(\tau) \approx i \int_{0}^{\infty e^{i \theta}} \exp \left(\frac{-z^{3}}{3}+i z \zeta\right) d z
$$

where $\cos 3 \theta>0$. There are three ranges of $\theta$, viz.

$$
\frac{-\pi}{6}+\frac{2 n \pi}{3}<\theta<\frac{\pi}{6}+\frac{2 n \pi}{3} \quad n=0,1,2 \text {, }
$$

which are admissible, and three corresponding functions $E_{n}(\zeta)$. The qeneral solution to $(I I .1)$ is a sum $r E_{0}+s E_{1}+(1-r-s) E_{2}$. A steepest descent calculation shows that only $E_{0}$ has the correct asymptotic behaviour. Thus, $r=1, s=0$, and the solution to Eq. (II.I) is

$$
E(\zeta)=-i \int_{0}^{\infty} \exp \left(\frac{-z^{3}}{3}+i z \zeta\right)
$$

To integräte $\operatorname{Im} E(\zeta)$, note that $\operatorname{Im} E(\zeta)$ is an even function of 5 . Hence, 
$-33-$

$$
\begin{aligned}
& \int_{-\infty}^{\infty} \operatorname{Im} E(\zeta) d \zeta=-2 \lim _{\mathbf{N} \rightarrow \infty} \int_{0}^{N} d \zeta \int_{0}^{\infty} \exp \left(-\frac{z^{3}}{3}\right) \cos z \zeta \\
& =-2 \lim _{\mathrm{N} \rightarrow \infty} \int_{0}^{\infty} \exp \left(-\frac{\mathrm{z}^{3}}{3}\right) \frac{\operatorname{sinNz}}{\mathrm{z}} \\
& =-\pi \text {. }
\end{aligned}
$$




\section{REFERENCES}

$I_{A}$. Vlasov, Zh. Eksp. Teor. Fiz. 8, 291-318 (1938).

2L. Landau, J. Phys. U. S. S. R. 10, 25 (1946); [zh. Eksp. Teor. Fiz. 16, 574 (1946)]:

${ }^{3}$ J. D. Jäekson, J. Nuclear Energy', Part. C: Plasma physics, $1,171(1960)$

4 John Dawson, Phys. Fluids 4 , 869 (1961).

$5_{K}$. M. Case, Ann. Phys. 7, 349 (1959).

6 N. G. Van Kampen, Physica 21 , 949 (1955).

7A. Lenäd and I. Bernstein, Phys. Rev. 112, 1456 (1958).

${ }^{8}$ C. H. Su and C. Oberman, Phys. Rev. Letters 20, 427 (1968).

${ }^{9}$ G. L. Johnston, Phys. Fluids 14, 2719 (1971):

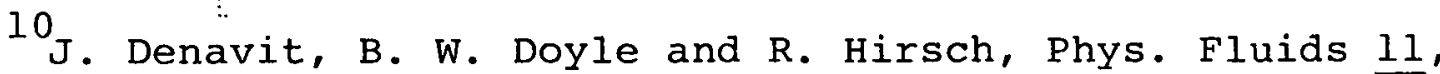
2241 (1968).

${ }^{11}$ C. T. Dum, Phys. Rev. Letters 35, 947 (1975).

12 This is just the real part of a wave with space-time dependence exp $-i[k x-(\omega-i \gamma) t]$. It is convenient to use real quantities to help keep track of phases.

13 V. E. Zakharov and V. I. Karpman, Sov. Phys. - JETP 16, 351 (1963); [Zh. Eksp. Teor. Fiz. 43, 490-499.(1962)].

14 This is proportional to $\mathrm{Hi}(i \xi)$. See p. 448 of Handbook of Mathematical Functions, edited by M. Abramowitz and I. Stegun (National Bureau of Standards Applied Mathematics Series, Number 55; $\ddot{U}$. S. Government Printing Office, Washington, D.C.) • 
15

This is only true because the slowing down term in the collision operator is negligible in the boundary layer.

${ }^{16}$ L. D. Landau and E. M. Lifshitz, Electrodynamies of Continuous Media (Addison-Wesley, Reading, Massachusetts, 1960), p. 256 .

17 T. E. Stringer, Phys. Fluids 13,810 (1970).

18 M. N. Rosenbluth, W. MacDonald, and D. Judd, Phys. Rev. 107,1 (1957).

${ }^{19}$ This is identical, except for a curious factor $3 / 2$, to the expression in reference 13. Their derivation is incomplete, however, for they do not mention the crucial equation, Eq. (I.6). I am indebted to Dr. E. Ott for a discussion concerning reference 8 . 


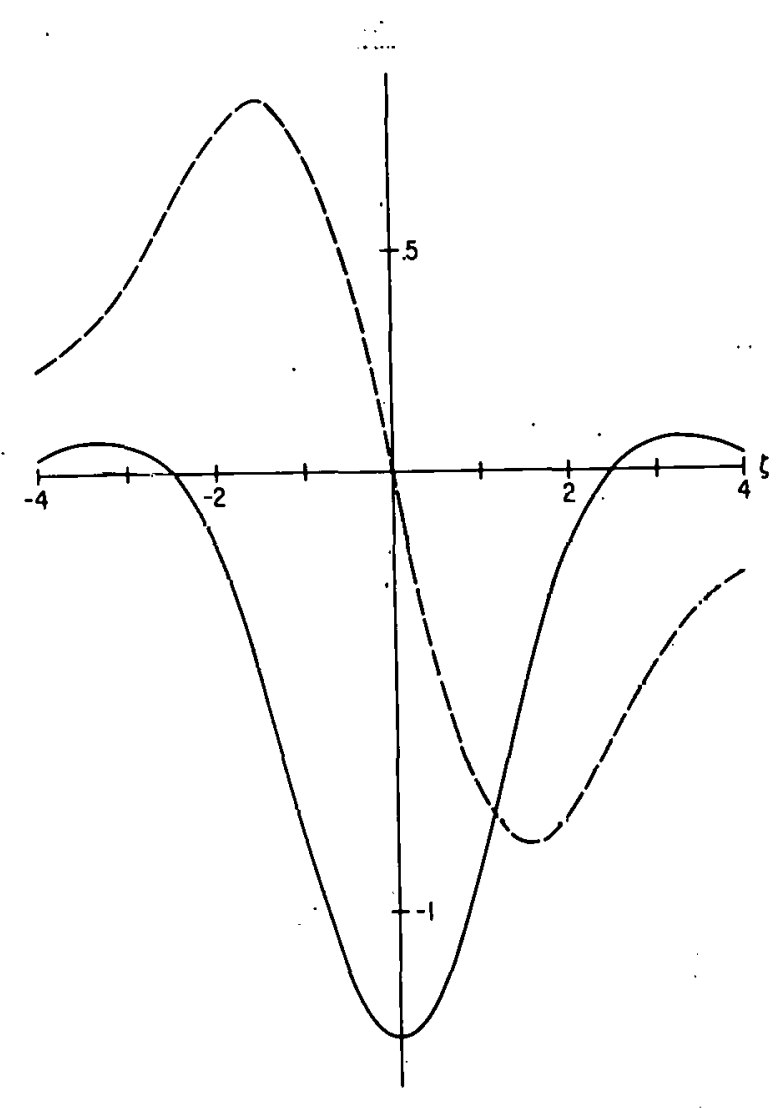

772006

Fig. 1. The functions a $(\zeta)$ (solid line) and $b(\zeta)$ (dashed line).

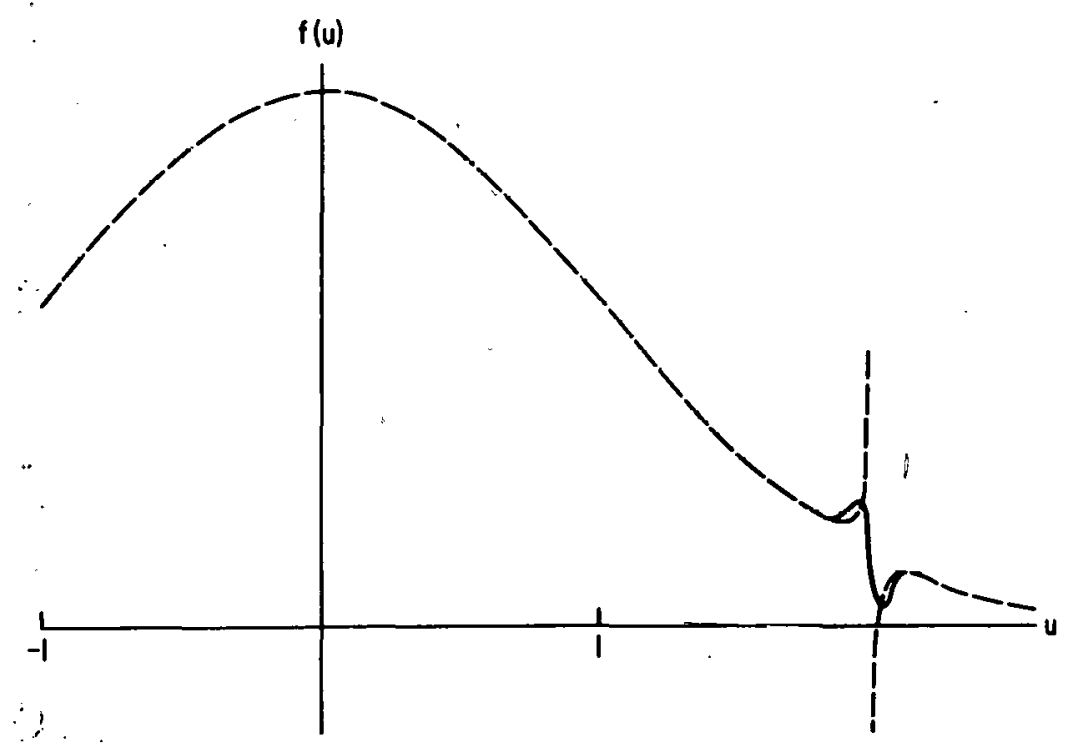

772010

Figure 2. Illustration of how the boundary layer behaviour of $b(\zeta)$ smoothly connects the collisionless regimes. The collisionless distribution $\mathrm{F}(\mathrm{u})$ is the dashed line. 

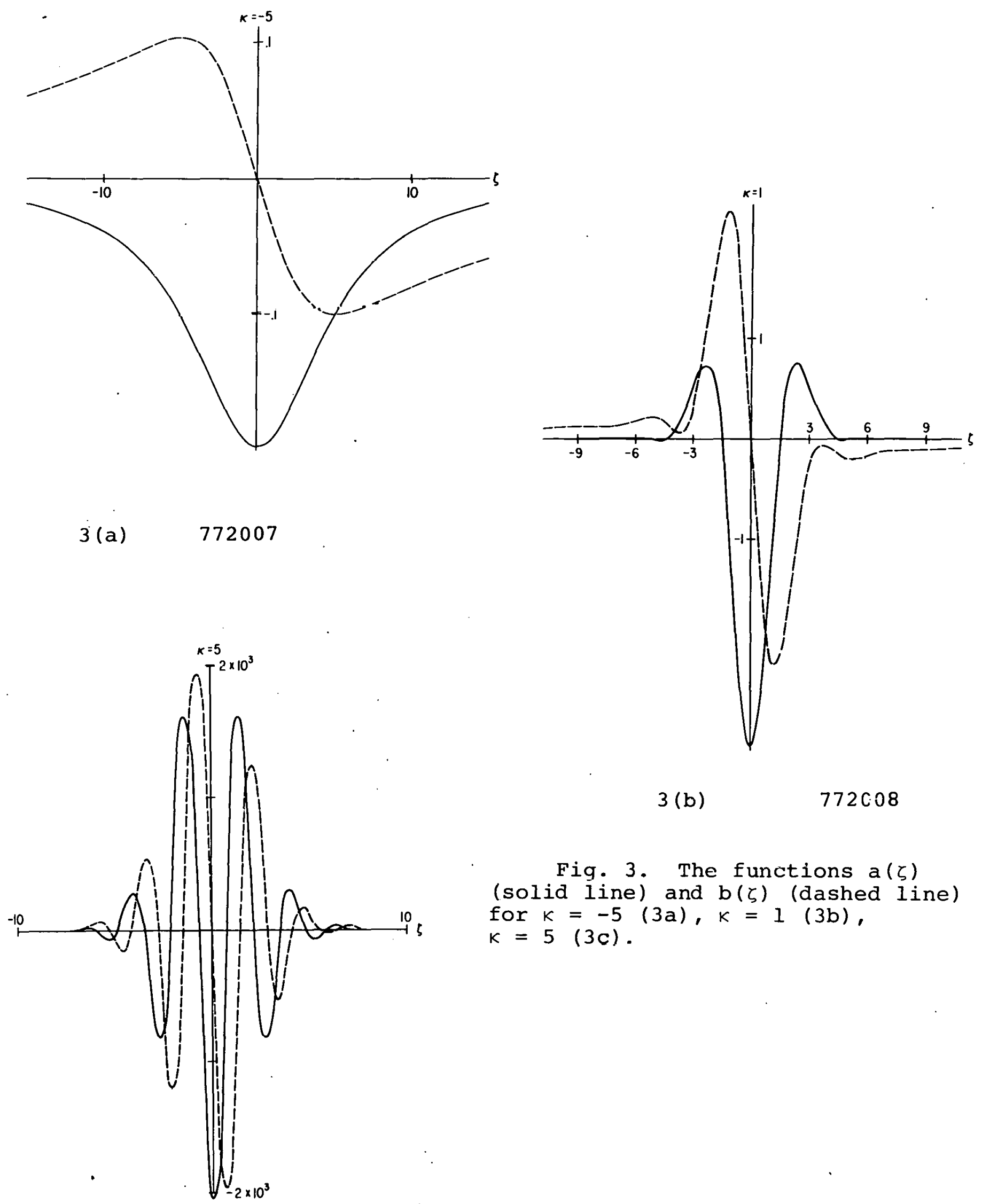

$772 C 08$

Fig. 3. The functions $a(\zeta)$ (solid line) and $b(\zeta)$ (dashed line) for $k=-5(3 a), k=1(3 b)$, $K=5(3 c)$. 


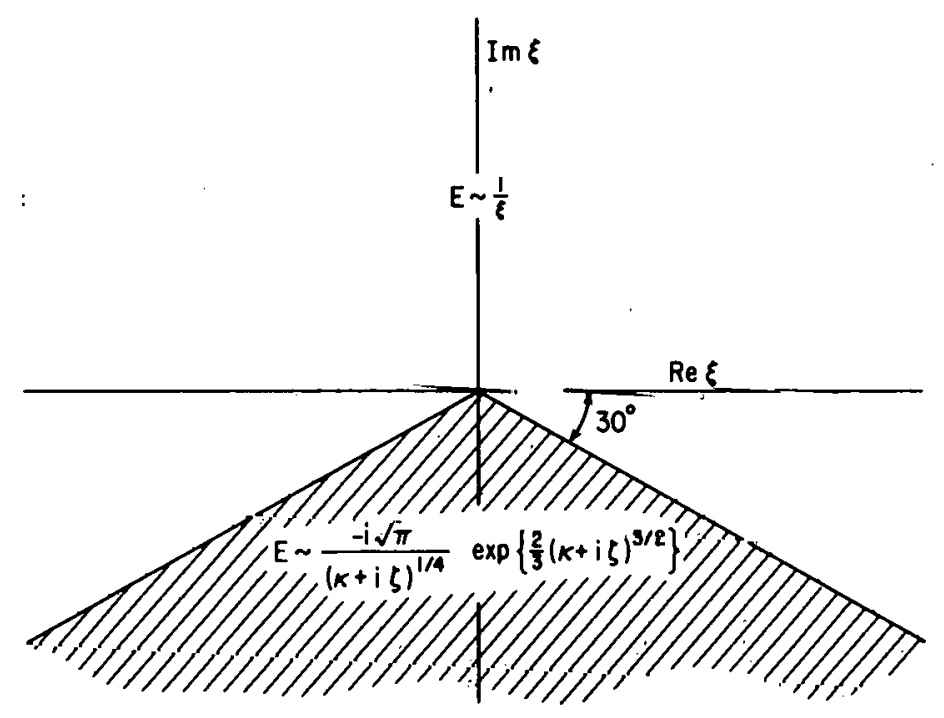

772012

Fig. 4. Asymptotic behaviour of $E(\xi)$.

1) 